\title{
UM SISTEMA DE ENRIQUECIMENTO SEMÂNTICO DE PERFIL DE USUÁRIO BASEADO EM TRAÇOS DIGITAIS PARA APOIO À APRENDIZAGEM INFORMAL NO CONTEXTO DA SAÚDE
}

\author{
Jonathan Darlan Cunegundes Moreira ${ }^{1}$ \\ Francisco Milton Mendes Neto ${ }^{1}$ \\ Alisson Alan Lima da Costa ${ }^{1}$ \\ Enio Lopes Sombra ${ }^{1}$ \\ Argemiro Soares de Aliança Neto ${ }^{1}$ \\ Ricardo Alexsandro de Medeiros Valentim² \\ ${ }^{1}$ Programa de Pós-Graduação em Ciência da Computação - PPGCC, Universidade Federal \\ Rural do Semi-Árido - UFERSA, Mossoró, Rio Grande do Norte, Brasil. \\ ${ }^{2}$ Universidade Federal do Rio Grande do Norte (UFRN), Natal, Rio Grande do Norte, Brasil. \\ \{jdarlan, miltonmendes, alisson, enio\}@ufersa.edu.br; argemironeto@petrobras.com.br; \\ ricardo.valentim@ufrnet.br.
}

Resumo. O processo de aprendizagem informal favorece a autoaprendizagem, relações sociais, o compartilhamento de experiências e a aprendizagem colaborativa. As tecnologias móveis podem auxiliar neste processo através de um ambiente ubíquo, promovendo o acesso ao conhecimento em qualquer lugar e a qualquer momento. Baseado no conceito de saúde 2.0, a ferramenta MobiLEHealth tem como objetivo proporcionar uma melhor qualidade de vida a pacientes em tratamento domiciliar, estimulando-os a tornam-se ativos nos cuidados relativos a sua saúde. Para isso promove um ambiente de aprendizagem informal através da recomendação personalizada de conteúdo a estes usuários, levando em consideração o seu cotidiano, monitorando-os por meio de tecnologias ubíquas. Para tornar isso possível é primordial conhecer o usuário e seus interesses sem a necessidade de sua intervenção. Este trabalho propõe um sistema de enriquecimento semântico de perfil de saúde do usuário para a ferramenta MobiLEHealth, que, através da análise de traços digitais, processamento de linguagem natural, ontologias e agentes de software, permite determinar a relação entre usuário, conteúdo e domínios relacionados à sua saúde.

Palavras-chaves: enriquecimento semântico, traços digitais, ontologias, aprendizagem informal, saúde 2.0.

\section{A SYSTEM OF SEMANTIC AUGMENTATION OF USER PROFILE BASED ON DIGITAL TRACES TO SUPPORT INFORMAL LEARNING IN THE HEALTH CONTEXT}

\begin{abstract}
The process of informal learning encourages self-learning, social relationships, sharing experiences and collaborative learning. Mobile technologies can assist in this process through a ubiquitous environment, promoting access to knowledge anywhere and anytime. Based on the concept of
\end{abstract}


health 2.0, the MobiLEHealth tool aims to provide a better quality of life to patients in home care, encouraging them to become active on health care. For this, promotes an environment of informal learning through personalized content recommendation, taking into consideration your daily life, tracking him with pervasive technologies. For this, it is essential to know the user and your interests without requiring your intervention. This paper proposes a system for semantic augmentation of user health profile for MobiLEHealth tool, which, by analysis of digital traces, natural language processing, ontologies and software agents, is able to determine the relationship between users, contents and domains related to their health.

Keywords: semantic augmentation, digital traces, ontologies, informal learning, health 2.0 .

\section{Introdução}

Muitas das doenças crônicas trazem limitações aos seus portadores, no entanto, boa parte delas pode ser prevenida, controlada e promovida uma melhor qualidade de vida aos seus portadores. $\mathrm{O}$ primeiro passo é conhecer a doença, contudo, isso implica que o paciente deve aceitar a doença e torna-se ativo nos cuidados relativos a ela.

O conceito de saúde 2.0 une aprendizagem informal ao uso de tecnologias e ferramentas da web 2.0 (redes sociais, registros pessoais de saúde, blogs, vídeos, serviços, etc.) como forma de capturar informações referentes à saúde do usuário, através da sua experiência. E pela combinação desses dados e informações, fornecer cuidados e conteúdos personalizados (Hughes et al., 2008). No entanto, essas informações não são fáceis de serem recuperadas, pois estão dispersas em diferentes sites e ferramentas e, na maioria das vezes, em linguagem natural. Além disso, mesmo após a captura desses dados, é necessário contextualiza-los a um domínio de conhecimento e fornecer relação com o usuário.

Diante deste cenário, este artigo apresenta um sistema para enriquecimento semântico do perfil de saúde do usuário, como parte de um modelo para apoiar a aprendizagem informal no contexto de saúde 2.0, o MobiLEHealth (Mendes Neto et al., 2014a). Este é destinado a pessoas portadoras de doença crônica e em tratamento domiciliar, promovendo o conhecimento sobre a doença e, consequentemente, uma melhoria na sua qualidade de vida (Mendes Neto et al., 2014b).

Através do processamento dos dados coletados pelo MobiLEHealth, oriundos de traços digitais do usuário, o sistema de enriquecimento propõe formar um perfil semântico do usuário capaz de determinar (i) os interesses do usuário relativo à sua saúde e (ii) a relação desses dados com domínios específicos. Os dados coletados passam por um processamento de linguagem natural e inferências sobre ontologias de domínio, gerando uma relação semântica entre esses dados e o usuário.

O perfil enriquecido do usuário irá fornecer ao MobiLEHealth informações necessárias para que sejam geradas recomendações de conteúdos personalizadas conforme as necessidades e interesses do usuário. 


\section{Aprendizagem Informal}

A aprendizagem formal ocorre sob a supervisão de um professor, enquanto que na aprendizagem informal o indivíduo é responsável pelo seu aprendizado, que ocorre através de informações cotidianas do seu ambiente (Jiugen et al., 2011). Essa forma de aprendizagem tem como principais características (i) a autonomia, onde o indivíduo é responsável por sua aprendizagem e determina o objetivo a ser alcançado, o conteúdo a ser abordado e como este será processado; (ii) o conhecimento, obtido por meio das interações sociais e profissionais; (iii) a diversidade, obtida através de experiências diárias, recursos do ambiente, bibliotecas, redes sociais, dentre outros.

As tecnologias móveis e a internet fornecem um ambiente favorável à aprendizagem informal. Elas eliminam a distância entre as pessoas no processo de comunicação, permitindo o compartilhamento de suas experiências diárias em qualquer lugar e a qualquer momento. Deste modo, estas tecnologias promovem a disseminação do conhecimento e a aprendizagem informal.

\section{Enriquecimento Semântico}

Segundo Redecker et al. (2010), tendências apontam que a mídia social irá ter um forte impacto sobre a aprendizagem informal, provendo conteúdo gerado pelo usuário por meio das experiências do mundo real. Porém, esses conteúdos geralmente não possuem um domínio de aprendizagem bem definido e normalmente são escritos em linguagem natural. Logo, para que a informação possa ser compreensível e tratável por um agente computacional, se faz necessário o uso de técnicas e ferramentas que proporcionem um entendimento mínimo sobre o conteúdo.

O enriquecimento semântico é o processo de anexar conceitos semânticos a partes específicas de um texto, provendo uma estrutura para interpretação automática de seu significado e possibilitando estender a compreensão do domínio de um determinado Traço Digital (TD) (Thakker et al., 2012). Para isso, é realizada a identificação e o mapeamento de termos-chave a partir do conteúdo textual através de técnicas e ferramentas de Processamento de Linguagem Natural (PLN), que analisam os radicais e origem das palavras e ajudam a determinar e encontrar as variações das mesmas, como conjugações de verbos, flexões de adjetivos, graus nos substantivos, etc. Além de encontrar e isolar frases das sentenças e períodos do texto (Russell and Norvig, 2009).

Os termos podem, então, ser semanticamente associados a conceitos oriundos de uma ontologia de domínio (Zapater and Mendes Neto, 2014), que especifica a conceitualização de um conhecimento, a fim de realizar uma marcação semântica (Karanasios et al., 2013). Por fim essas marcações são vinculadas ao usuário, permitindo traçar o seu perfil e realizar a categorização dos conteúdos automaticamente.

\subsection{Enriquecimento Semântico de Perfil de Usuário}

Recomendar conteúdo personalizado requer conhecer a quem está recomendando e, baseado nas suas necessidades, preferências, interesses, características sociais e psicológicas, direcionar conteúdo relevante (Shen et al, 2005). Para isso é determinante 
identificar o usuário e manter um perfil a ele associado. Porém nem sempre é trivial manter essa estrutura e utilizar coerentemente estes dados.

As ontologias contribuem neste sentido, permitindo criar perfis semânticos de usuários, modelando seus conceitos e relacionamentos, e possibilitando realizar inferências sobre eles (Heckmann et al., 2005). As ontologias de perfis de usuários mantêm relações semânticas entre usuário, conteúdo e conceitos de domínio, isolandoas das ontologias de domínio.

\section{Trabalhos Relacionados}

O enriquecimento semântico é um tema bastante abordado em pesquisas acadêmicas. Ji et al. (2013) propõem um sistema semântico de informações médicas que coleta informações na internet, converte em conhecimento para a ontologia e responde a perguntas dos usuários.

Baseado nos registros eletrônicos de saúde e bases de conhecimentos, Camous et al. (2008) propõem, através do uso de ontologias e da análise do perfil do usuário, validar o consumo de medicamentos (automedicamento) por parte do paciente como apoio ao sistema XIMSA.

O presente trabalho propõe um sistema, integrado ao MobiLEHealth, de enriquecimento semântico do perfil de saúde do usuário, considerando a sua condição de saúde e as suas interações cotidianas, por meio de seus traços digitais. O sistema permite determinar os interesses do usuário e a relação entre conteúdo e domínios, de forma ubíqua e implícita.

\section{MobiLEHealth}

O MobiLEHealth propõe o fornecimento de conteúdos personalizados e adaptados a pessoas portadoras de doenças crônicas, através da aprendizagem contínua no dia-a-dia, visando à obtenção de um maior conhecimento sobre a doença e melhoria da qualidade de vida.

Como citado anteriormente, o sistema proposto é parte da arquitetura do MobiLEHealth que é apresentada na Figura 1. O modelo abstrato mostra a comunicação entre os componentes da arquitetura, que está organizada em cinco componentes distintos: (i) Serviços Web, (ii) Dispositivo Móvel, (iii) Enriquecimento Semântico, (iv) Repositório Semântico e (v) Recomendação de Conteúdo, e envolve três áreas de conhecimento principais: (i) monitoramento contínuo de usuários, (ii) enriquecimento semântico de perfil de usuário e de conteúdos, e (iii) recomendação inteligente de conteúdos.

A arquitetura foi projetada de forma modular, delegando atividades e funções específicas para cada um dos componentes. Visando manter a consistência e dar inteligência ao sistema, os componentes são coordenados por um sistema multiagente (SMA) do tipo comunidade de especialistas (Belifemine et al., 2007), onde os seis agentes encontram-se no mesmo nível e são especialistas em determinada tarefa: (i) o Agente de Interface é responsável pelas ações que exigem interação com o usuário, (ii) 
o Agente ODL é responsável pelo monitoramento e a captura de informações contextuais do usuário, (iii) o Agente Analisador de Conteúdo é responsável pelo processamento do TD, através de PLN e outros algoritmos, transformando-o em um formato interpretável pelo processador semântico, (iv) o Agente de Enriquecimento Semântico é responsável pelo processamento do enriquecimento semântico do conteúdo e do perfil do usuário, (v) o Agente recomendador é responsável pela seleção dos conteúdos adequados a serem recomendados, e o (vi) Agente de Controle desempenha a função de directory facilitator (DF), fornecendo uma arquitetura do tipo "quadro-negro" mediando a comunicação entre os agentes.

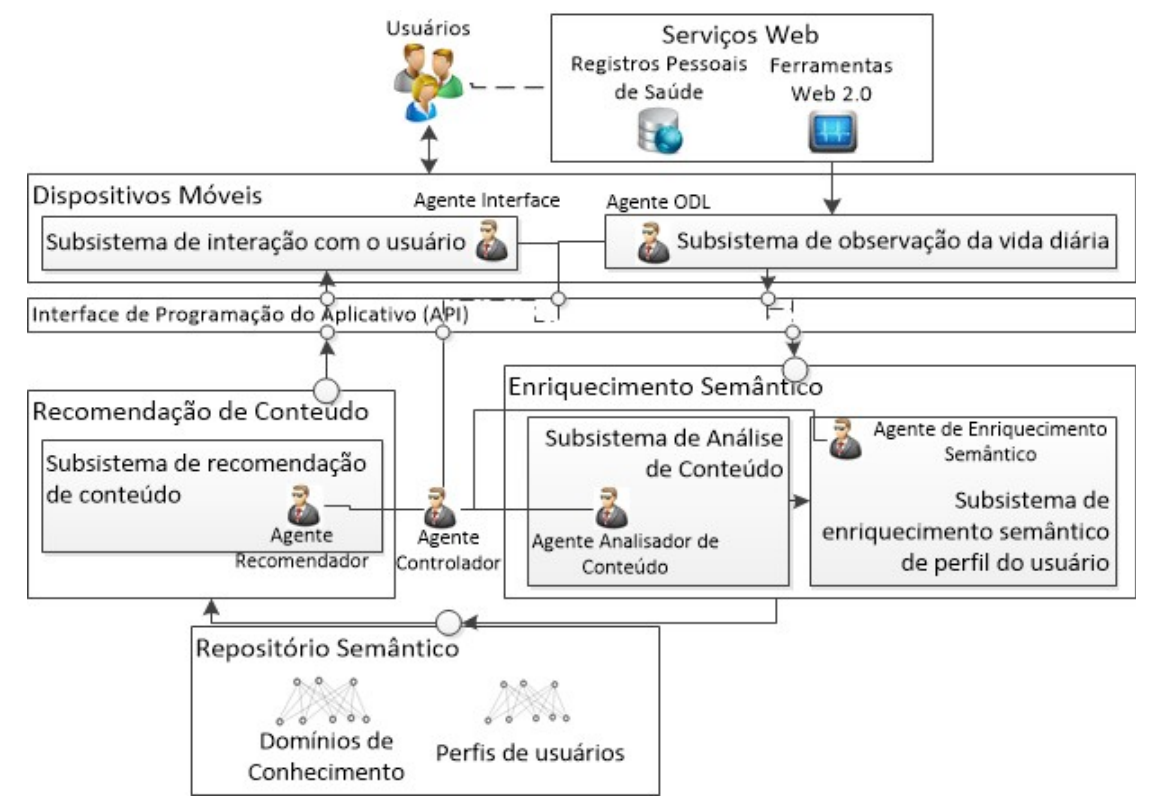

Figura 1 - Modelo da Arquitetura do MobiLEHealth (Mendes Neto et al., 2014a)

\section{Sistema de Enriquecimento Semântico Proposto}

O Sistema de Enriquecimento Semântico tem a tarefa de receber os conteúdos coletados e enriquecer semanticamente o perfil de usuário com base nos domínios de conhecimentos registrados no repositório semântico, através de técnicas de processamento de linguagem natural, ontologias e algoritmos de seleção e marcação de conteúdo. O sistema apresenta uma arquitetura modular fornecendo uma interface padrão que o integra ao MobiLEHealth de forma coesa, isolando o componente e garantindo a consistência do seu funcionamento.

Os elementos da arquitetura estão representados pela Figura 2 e serão descritos a seguir. O Pre-Processador realiza o carregamento e um tratamento prévio do recurso que será enriquecido, verificando a integridade e a conformidade com os tipos de arquivos e conteúdos suportados pelo sistema.

O Analisador Semântico realiza um processamento de linguagem natural no conteúdo, identificando e isolando anotações e marcações já existentes, como marcações HTML e XML. O conteúdo restante é analisado morfologicamente e sintaticamente, isolando os tipos de palavras, suas flexões, radicais e funções semânticas. Com base nestes dados, uma busca por termos semelhantes nas ontologias de domínio é realizada e as correspondências encontradas são armazenadas em 
estruturas de dados específicas. Essa estrutura de dados é enviada para o Indexador Semântico, que trata e armazena as informações do enriquecimento nas ontologias de perfil e domínio.

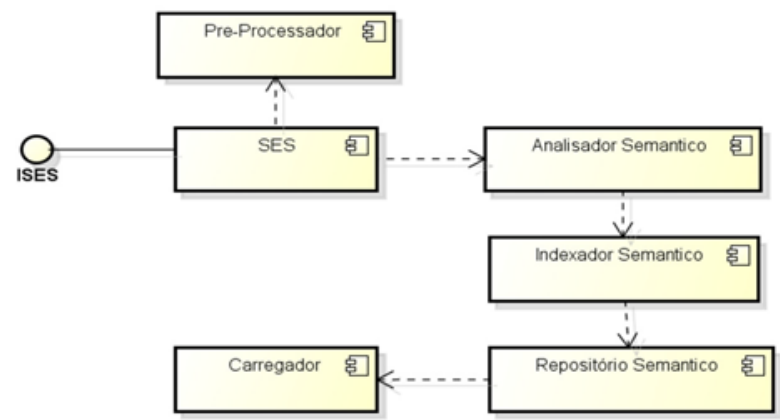

Figura 2 - Arquitetura do Sistema de Enriquecimento Semântico

O Repositório Semântico fornece ontologias de domínio, que representam a modelagem dos conceitos relacionados à saúde que compõem o sistema, e as ontologias de perfil, que representam os usuários ou os conteúdos. Essa separação entre a ontologia de domínio e de perfil é outro fator importante da arquitetura, pois visa prover a portabilidade, já que as ontologias de domínio podem ser utilizadas por outras aplicações, e a expansibilidade, pois novos domínios relacionados à saúde podem ser incluídos e o perfil do usuário pode ser modelado sem precisar de alterações no sistema.

\section{Resultados e Discussão}

Para os experimentos de validação do sistema foram selecionados como domínios da aplicação as doenças crônicas Diabetes e a Esclerose Lateral Amiotrófica (ELA). Estas doenças foram escolhidas (i) pela abrangência em que a doença atinge a população, no caso da Diabetes, (ii) pelo impacto que causam no cotidiano das pessoas portadoras, no caso da ELA, e (iii) pelo fato de que a falta de informação dificulta ainda mais a convivência com essas doenças. Outro ponto levado em consideração foi o fato do Laboratório de Inovação Tecnológica em Saúde (LAIS) do Hospital Universitário Onofre Lopes (HUOL), cujo coordenador faz parte da equipe deste projeto, trabalhar com estas duas doenças, provendo os conhecimentos necessários para construção das ontologias de domínio.

O processo de validação passou por quatro etapas: (i) a primeira foi a criação das ontologias de domínio para realizar o processo de enriquecimento semântico, (ii) em seguida houve a seleção dos conteúdos e o processamento semântico; (iii) a terceira etapa foi a definição e geração dos perfis de usuários; e, por fim, (iv) foi realizada a análise dos resultados.

\section{A. Ontologias}

Foram criadas duas ontologias de domínio, uma para diabetes e outra para ELA, que descrevem os conceitos de cada uma das doenças crônicas. Para a criação foram selecionados alguns conteúdos de portais conceituados sobre os domínios em questão como, por exemplo, os portais da International Diabetes Federation (IDF) (www.idf.org) e da ALS Association (ALSA) (www.alsa.org). Desses conteúdos foram 
extraídos termos comuns em mais de 90\% dos conteúdos selecionados e categorizados para a criação da ontologia. Desse processo foram selecionados 25 (vinte e cinco) termos para o domínio diabetes e 51 (cinquenta e um) termos para o domínio ELA, classificados em 16 (dezesseis) categorias, que para os domínios selecionados foram definidos de forma idêntica. Essas categorias podem ser vistas na Figura 3.

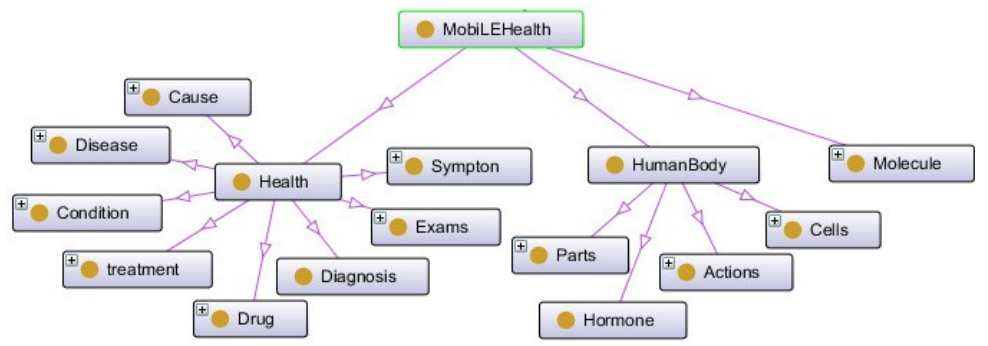

Figura 3 - Estrutura de classes das ontologias de domínio do MobiLEHealth.

\section{B. Conteúdos}

Para realizar o enriquecimento semântico, foram selecionados 80 (oitenta) conteúdos de cada um dos domínios. A seleção ocorreu pela internet e de forma manual, com o intuito de garantir (i) que o conteúdo era fortemente relacionado ao domínio e (ii) a qualidade e confiabilidade do conteúdo. Além disso, foram selecionados aleatoriamente 80 (oitenta) conteúdos de assuntos diversos, como economia, esporte, jogos, etc., pois acessos a conteúdos diversos fazem parte do cotidiano dos usuários.

Após a análise semântica dos 240 (duzentos e quarenta) conteúdos, foi verificado o percentual de acerto das anotações semânticas. Uma anotação é considerada "certa” se fizer referência à ontologia de domínio correlata ao assunto do conteúdo. Qualquer anotação semântica gerada para um conteúdo de assuntos diversos é considerada “errada”. Como resultado obteve-se uma precisão média de 75,91\% com um desvio padrão de 6,035\%. Esses dados podem ser observados na Tabela 1.

Tabela 1 - Percentual de acertos por domínio

\begin{tabular}{|l|l|l|l|}
\hline Domínio & Qtd Certos & Errados & \% Acerto \\
\hline Diabetes & 17.281 & 3.818 & $81,94 \%$ \\
\hline ELA & 8.662 & 3.735 & $69,87 \%$ \\
\hline Média & 25.943 & 7.553 & $75,91 \%$ \\
\hline
\end{tabular}

\section{Perfis de usuários}

Os perfis de usuários foram definidos baseados nas possibilidades de interesses dos usuários considerando os três assuntos dos conteúdos (diabetes, ELA e diversos), onde foram definidos quatro perfis: (i) diabetes, (ii) ELA, (iii) diabetes e ELA, (iv) nenhum.

Para cada perfil foram simulados 30 (trinta) usuários e para cada usuário foram realizados 20 (vinte) acessos a conteúdos, distribuídos conforme demonstrado na Tabela 2. Ao todo foram simulados 120 (cento e vinte) perfis de usuários e 2.400 (dois mil e quatrocentos) acessos a conteúdos.

Tabela 2 - Distribuição em percentual dos acessos para cada perfil dos dados simulados

\begin{tabular}{|l|l|l|l|}
\hline Perfil & Diabetes & ELA & Diversos \\
\hline
\end{tabular}




\begin{tabular}{|c|c|c|c|}
\hline \multicolumn{2}{|c|}{ CINTED-UFRGS } & \multicolumn{2}{|c|}{ Novas Tecnologias na Educação } \\
\hline Diabetes & $70 \%$ & $10 \%$ & $20 \%$ \\
\hline ELA & $10 \%$ & $70 \%$ & $20 \%$ \\
\hline Diabetes e ELA & $40 \%$ & $40 \%$ & $20 \%$ \\
\hline Nenhum & $15 \%$ & $15 \%$ & $70 \%$ \\
\hline
\end{tabular}

\section{Resultados}

Para avaliar a eficácia do sistema de enriquecimento semântico foram realizadas (i) a validação do índice de relação do conteúdo com o domínio e (ii) a validação do índice de interesse do usuário.

Em ambos os casos citados a cima, o cálculo da eficácia considera que os índices abaixo de $30 \%$ representam a não existência de relação do conteúdo ou usuário com o domínio. A variação de $30 \%$ a $100 \%$ representa nível de relação com o domínio e será utilizada pelo sistema de recomendação do MobiLEHealth para selecionar os conteúdos mais apropriados ao usuário.

Para analisar os resultados foram consideradas as métricas verdadeiro positivo (VP) e verdadeiro negativo (VN), onde VP são os índices que submetidos a um domínio tiveram os valores na margem esperada, enquanto que VN são os índices que não retornaram valores dentro da margem esperada. O índice de eficácia (Ei) é avaliado pela representatividade do VP, como mostrado na Equação 1.

$$
\mathrm{Ei}=\frac{\sum \mathrm{VP}}{\sum(\mathrm{VN}+\mathrm{VP})}
$$

Equação 1 - Cálculo do índice de eficácia (Ei).

Foi avaliada a relação recurso-domínio para cada um dos 240 (duzentos e quarenta) conteúdos para os dois domínios (diabetes e ELA). Considerando a metodologia de avaliação descrita anteriormente, foi obtida uma eficácia de 91,42\% do sistema de enriquecimento semântico ao determinar a qual domínio um conteúdo pertence. Os detalhes podem ser observados na Figura 4.

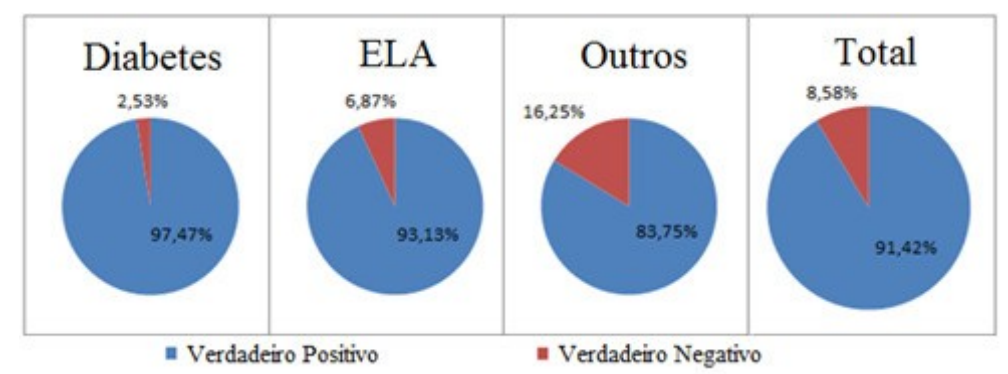

Figura 4 - Resultado da análise dos índices da relação do conteúdo com o domínio.

Na validação do índice de interesse do usuário, foi analisado cada usuário para cada um dos domínios e foi obtida uma eficácia de $90 \%$ ao determinar qual o domínio de interesse do usuário. O detalhamento desses resultados pode ser observado na Figura 5 .

Como pôde ser comprovado pelos índices de eficácia, o sistema de recomendação apresentou resultados satisfatórios ao determinar a relação de um conteúdo com um domínio e o interesse do usuário em um domínio. Porém percebeu-se 
que a precisão semântica depende das ontologias de domínio, que precisam ser bem definidas.

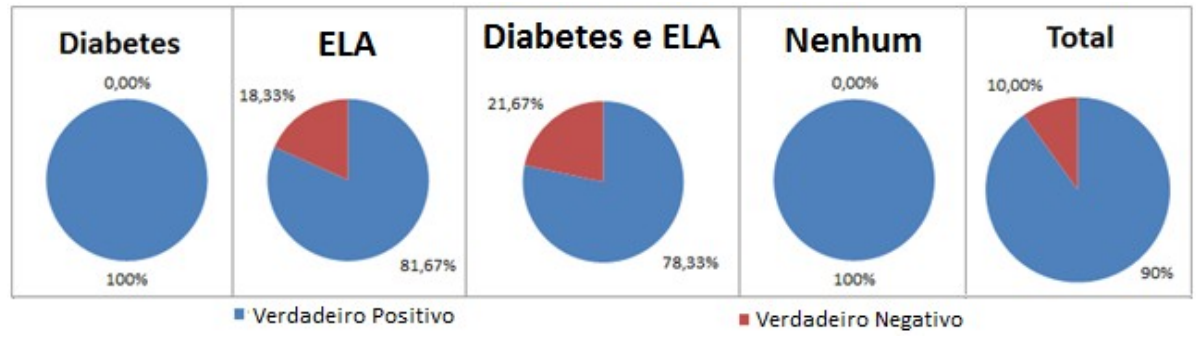

Figura 5 - Resultado da análise dos índices de domínio de interesse do usuário.

A separação do perfil do usuário das ontologias de domínio foi outro ponto relevante, uma vez que se mostrou eficaz e flexível, permitindo a extensão da aplicação a outros domínios sem a necessidade de alterações no código do sistema.

\section{Conclusões}

Neste artigo foi apresentado um sistema para enriquecimento semântico do perfil de saúde do usuário para apoiar a aprendizagem informa no contexto da saúde. O sistema proposto faz parte da ferramenta MobiLEHealth, e visa apoiar o componente de recomendação personalizada de conteúdos na seleção dos conteúdos que mais se adequam às necessidades e interesses dos usuários.

Através do uso de técnicas de PLN e ontologias, o sistema implementado provê um perfil semântico de usuário capaz de determinar (i) o índice de relação de interesse de um usuário com um domínio e (ii) o índice de relação de um conteúdo com um domínio, baseado em ontologias de domínio.

Os resultados demonstraram que é possível definir os interesses do usuário por meio de perfis semânticos, relacionados a domínios de conhecimentos, gerados a partir de sua experiência e informações referentes à sua saúde, através do monitoramento do seu contexto diário.

Como trabalhos futuros, pretende-se realizar um estudo de caso real com usuários portadores de doenças crônicas, a fim de comprovar que os índices fornecidos pelo sistema proposto possibilitam uma melhor eficácia na recomendação de conteúdos personalizados pelo MobiLEHealth e, principalmente, aprendizagem sobre saúde pessoal de forma flexível e ubíqua.

\section{Referências bibliográficas}

BELlifEMINE, F. L.; CAIRE, G.; GREENWOOD, D. Developing Multi-Agent Systems with JADE. 1 edition ed. Hoboken, NJ: Wiley, 2007.

CAMOUS, F.; MCCANN, D.; ROANTREE, M. Capturing Personal Health Data from Wearable Sensors. In: INTERNATIONAL SYMPOSIUM ON APPLICATIONS AND THE INTERNET, 2008. SAINT 2008, jul. 2008. p. 153-156. 
HECKMANN, D.; SCHWARTZ, T.; BRANDHERM, B.; SCHMITZ, M.; VON WILAMOWITZ-MOELLENDORFF, M. Gumo: The General User Model Ontology. UM’05, 2005, Berlin, Heidelberg: Springer-Verlag, 2005. p. 428-432.

HUGHES, B.; JOSHI, I.; WAREHAM, J. Health 2.0 and Medicine 2.0: Tensions and Controversies in the Field. Journal of Medical Internet Research, v. 10, n. 3, p. e23, 6 ago. 2008.

JI, J.; CHEN, L.; ZHAO, Q. Semantic medical system for health condition awareness. In: 2013 IEEE INTERNATIONAL CONFERENCE ON CYBERNETICS (CYBCONF), jun. 2013. p. 225-230.

JIUGEN, Y.; RUONAN, X.; XIAOQIANG, H. Constructing informal learning mode based on social software. In: 2011 6TH INTERNATIONAL CONFERENCE ON COMPUTER SCIENCE EDUCATION (ICCSE), ago. 2011. p. 1227-1230.

KARANASIOS, S.; THAKKER, D.; LAU, L.; ALLEN, D.; DIMITROVA, V.; NORMAN, A. Making sense of digital traces: An activity theory driven ontological approach. Journal of the American Society for Information Science and Technology, v. 64, n. 12, p. 2452-2467, 1 dez. 2013.

MENDES NETO, F. M.; DA COSTA, A. A. L.; SOMBRA, E. L.; MOREIRA, J. D. C.; VALENTIM, R. A. M.; SAMPER, J. J.; DO NASCIMENTO, R. P. C.; FLORES, C. D. Content's Personalized Recommendation for Implementing Ubiquitous Learning in Health 2.0, Latin America Transactions, IEEE (Revista IEEE America Latina), 2014 (Accepted for Publication).

MENDES NETO, F. M.; DA COSTA, A. A. L.; SOMBRA, E. L.; MOREIRA, J. D. C.; VALENTIM, R. A. M.; SAMPER, J. J.; DO NASCIMENTO, R. P. C.; FLORES, C. D. An approach for recommending personalized contents for homecare users in the context of health 2.0, in 2014 7th Euro American Conference on Telematics and Information Systems (EATIS), 2014.

REDECKER, C.; ALA-MUTKA, K.; PUNIE, Y. Policy Brief: Learning 2.0 - The Impact of Social Media on Learning in Europe. [S.l.], 2010. JRC 56958. Disponível em: $<$ http://ftp.jrc.es/EURdoc/JRC56958.pdf>. Acesso em: 12 dez 2013.

RUSSELL, S.; NORVIG, P. Artificial Intelligence: A Modern Approach. 3 edition ed. Upper Saddle River: Prentice Hall, 2009.

SHEN, X.; TAN, B.; ZHAI, C. Implicit User Modeling for Personalized Search. CIKM '05, 2005, New York, NY, USA. New York, NY, USA: ACM, 2005. p. 824-831.

THAKKER, D.; DESPOTAKIS, D.; DIMITROVA, V.; LAU, L.; BRNA, P. Taming Digital Traces for Informal Learning: A Semantic-driven Approach. in Proceedings of the 7th European Conference on Technology Enhanced Learning, Berlin, Heidelberg: Springer-Verlag, 2012. p. 348-362.

ZAPATER, J. J. S.; MENDES NETO, F. M. . Uso de Tecnologías Semánticas en Diferentes Dominios de Aplicación: Entorno Educativo y Sistemas de Información de Tráfico Vial. 1. ed. EAE - Editorial Academica Espanola, 2014. 330p. 